\title{
Management of the post-bariatric surgery patient - what an internist needs to know
}

\author{
Gabriela Handzlik-Orlik ${ }^{1}$, Michał T. Holecki ${ }^{1}$, Leszek Kędzierski ${ }^{1}$, Mariusz Wyleżoł², Jan Duława ${ }^{1}$ \\ ${ }^{1}$ Department of Internal Medicine and Metabolic Diseases, Medical University of Silesia, Katowice, Poland \\ 2Department of Surgery, Military Institute of Aviation Medicine, Warsaw, Poland
}

\begin{abstract}
Bariatric surgery is the most effective method of achieving long-lasting weight loss and improving metabolic and cardiovascular outcomes in selected obese patients (i.e. with body mass index [BMI] $\geq 40 \mathrm{~kg} / \mathrm{m}^{2}$ without coexisting morbidities or BMI $\geq 35 \mathrm{~kg} / \mathrm{m}^{2}$ with severe obesity-related diseases). As the epidemic of obesity grows, it is estimated that by 2030 around $11 \%$ of the population will be severely obese. Therefore, the number of bariatric procedures performed increases year after year, and it reached 179,000 in 2013. Bariatric procedures can be divided into three main types: restrictive, malabsorptive, and mixed procedures. While, careful multi-disciplinary preoperative assessment of the patient is an inseparable part of bariatric procedures, there is also a need for multi-disciplinary management after bariatric surgery. This should include surveillance by an internist, surgeon and registered dietician. However, treatment outcome is primarily dependent on the patient's compliance and awareness of the need for regular follow-up. This cannot be achieved without proper behavioral and psychological support, as it is as vital as other surveillance aspects. In this review we discuss early and late complications of bariatric surgery, its influence on obesity-related diseases, and changes in body composition and metabolism. According to recent guidelines and recommendations of American and European medical societies, we summarize available standards of post-bariatric management.
\end{abstract}

Key words: bariatric surgery, obesity, dumping syndrome, diabetes mellitus, lipid metabolism.

\section{Introduction}

Obesity is one of the most alarming health care problems worldwide, since around 2 billion adults are considered to be overweight (body mass index [BMI] between 25 and $\left.30 \mathrm{~kg} / \mathrm{m}^{2}\right)$ or obese $\left(\mathrm{BMI} \geq 30 \mathrm{~kg} / \mathrm{m}^{2}\right)[1]$. The steadily growing prevalence of obesity is a matter of common concern, and forecasts predict that $11 \%$ of the population will be severely obese (BMI $\left.\geq 40 \mathrm{~kg} / \mathrm{m}^{2}\right)$ by 2030 [2]. Overweight and obesity are major risk factors of chronic diseases, such as cardiovascular disease, type 2 diabetes mellitus (T2DM), some types of neoplasms and musculoskeletal disorders.

Non-surgical management of obesity is considered as a first line therapy, and it includes lifestyle changes (increased physical activity, modification of diet and behavior) and pharmacological treatment. It can induce mean weight losses of $5-10 \%$. However, long-term weight loss maintenance is usually poor or insufficient. For patients who did not succeed in non-surgical intervention, but are at increased risk of obesity-related mortality and/or meet the criteria for surgery, a bariatric procedure may be considered.

The number of bariatric surgery procedures performed in the U.S. reached 173000 in 2012, and 179000 in 2013. This large group of patients will require lifelong, regular, qualified, multidisciplinary surveillance including medical, surgical, behavioral and dietary observation.

\section{Bariatric surgery}

\section{Bariatric surgery techniques}

Bariatric surgical procedures can be divided into three main types: restrictive, malabsorptive, and mixed procedures. Adjustable gastric banding and sleeve gas- 
trectomy are predominantly restrictive procedures. They aim at reduction of oral intake by limiting gastric volume and production of early satiety; they leave the alimentary canal in continuity, minimizing the risk of metabolic complications. Bilio-pancreatic diversion as a malabsorptive procedure leads to reduction of stomach size and the physiological condition of malabsorption. Mixed procedures, such as gastric bypass and sleeve gastrectomy with duodenal switch, apply both techniques simultaneously [3].

The most commonly performed procedures are Rouxen-Y gastric bypass (RYGB), bilio-pancreatic diversion (BPD), sleeve gastrectomy (SG) and adjustable gastric banding (AGB) [4]. Bariatric surgery results in significant long-lasting weight loss, improves obesity-related co-morbidities and decreases mortality [5]. It must be mentioned that bariatric procedures (especially RYGB and SG) lead to gut hormone changes that may be implicated in these beneficial effects [6].

\section{Indications for bariatric surgery}

According to the guidelines of the National Institute of Clinical Excellence, the European Association for the Study of Obesity and the American Association of Clinical Endocrinologists, bariatric surgery should be considered in patients with:

- severe obesity $\left(\mathrm{BMI} \geq 40 \mathrm{~kg} / \mathrm{m}^{2}\right)$ without coexisting obesity related disease,

- moderate obesity (BMI $\geq 35 \mathrm{~kg} / \mathrm{m}^{2}$ ) with severe obesityrelated conditions such as type 2 diabetes mellitus, cardiovascular disease or obstructive sleep apnea $[7,8]$.

Furthermore, patients with BMI of $30-34.9 \mathrm{~kg} / \mathrm{m}^{2}$ with T2DM and other obesity related diseases may also be offered a bariatric procedure, although evidence of longterm benefit in these patients is still limited.

\section{Influence of bariatric surgery on obesity- related diseases}

Compared to conventional treatment of obese patients, bariatric surgery is associated with a highest long-term reduction in all-cause mortality [9-11] and decreased prevalence and incidence of type 2 diabetes, myocardial infarction, stroke and cancer [12].

\section{Type 2 diabetes mellitus}

Recent emerging data on bariatric procedures indicate that bariatric surgery might be considered as metabolic surgery. This term refers to procedures intended to treatment of T2DM and to reduce cardiometabolic risk factors. According to the International Diabetes Federation, patients with $\mathrm{BMI} \geq 35 \mathrm{~kg} / \mathrm{m}^{2}$ and T2DM who are inadequately controlled by lifestyle and medical therapy should be considered for bariatric surgery [9]. This statement is supported by a number of recent findings indicating significant benefits after a bariatric procedure in type 2 diabetic patients, including diabetes remission (albeit defined by varying criteria across different studies) in up to $72 \%$ of patients 2 years after surgery $[12,13]$.

The remission of T2DM is observed within several days or weeks of the procedure, long before the reduction of body mass is noticeable. However, the improvement in metabolic condition may be transient, and within 3 years after surgery, $24 \%$ of patients who initially improve relapse into T2DM [14], and only $36 \%$ remain free of diabetes after 10 years [11]. Nevertheless, a long-term prospective controlled trial provided data confirming the efficacy of bariatric surgery on T2DM, with diabetes remission rates increased several-fold at 2 years (odds ratio $\mathrm{OR}=8.42, p<0.001)$ and 10 years $(\mathrm{OR}=3.45$, $p<0.001$ ) [14] compared to conventional treatment.

\section{Cardiovascular disease and hypertension}

Obesity is associated with cardiomyopathy, left ventricular hypertrophy and diastolic dysfunction. Increased circulatory volume and cardiac output may also lead to the development of atrial dilatation and systolic dysfunction [4]. Bariatric procedures lead to a $50 \%$ lower incidence of fatal and total cardiovascular events (after adjustment for age and BMI) compared to non-surgical treatment [5]. Bariatric surgery beneficially modulates and reverses ventricular remodeling, improving both systolic and diastolic function [15]. The mechanisms are not fully elucidated, but improvements in hyperinsulinemia, dyslipidemia and hypertension are believed to facilitate the process. The mechanical weight loss effect and decreased inflammation may also be pivotal.

Initial improvements in hypertension and dyslipidemia may diminish with time; however, after 10 years of follow-up blood pressure and serum total cholesterol are still reduced compared to baseline values [16]. According to a systematic review and meta-analysis by Buchwald et al., $78.5 \%$ of patients show postoperative resolution or improvement of hypertension. Similarly, hyperlipidemia (particularly total cholesterol, low density lipoproteins and triglycerides) is improved in approximately $70 \%$ of patients, with no influence on high-density lipoproteins [16].

\section{Kidney disease}

Clinical evidence suggests that weight loss has beneficial effects on renal function [17]. Bariatric surgery improves the glomerular filtration rate (GFR) in patients with pre-existing mild chronic kidney disease (CKD) [18] and reduces albuminuria and/or proteinuria 
in patients with T2DM [19]. A small retrospective study by Navaneethan et al. [18] showed that mean GFR after 6 months of follow-up had improved from $47.9 \mathrm{ml} / \mathrm{min} / 1.73 \mathrm{~m}^{2}$ before the procedure to $56.6 \mathrm{ml} /$ $\min / 1.73 \mathrm{~m}^{2}(p<0.001)$ and after 12 months GFR increased to $61.6 \mathrm{ml} / \mathrm{min} / 1.73 \mathrm{~m}^{2}(p<0.001)$. Nevertheless, there is as yet no consensus on the effect of bariatric procedures on renal function, mainly due to difficulties in estimation of GFR in this group of patients (common equations are restricted by body size confounders).

Furthermore, bariatric surgery may be associated with acute kidney injury (AKI) and severe electrolyte disturbances, particularly after RYGB and BPD. The etiology of renal failure after these procedures is complex and may include dehydration due to diarrhea or vomiting [20], and intratubular calcium oxalate crystal deposition [21]. Post-bariatric AKI can develop in up to $8.6 \%$ of patients, mainly during the first 72 hours after the surgery. Significantly, the incidence of AKI after bariatric surgery is much greater than that reported after any other general surgical procedures [22].

\section{Other disorders}

Obese individuals have an increased prevalence of obstructive sleep apnea (OSA). Almost 70\% of patients referred for bariatric surgery suffer from this disorder, and more than $40 \%$ fulfill the criteria of severe OSA [23]. Bariatric surgery can produce improvement or even full remission of OSA in nearly $84 \%$ of patients (95\% CI: 71.8-95.4\%) [16], an improvement that is maintained in the long term. The mechanisms are not fully established but probably relate to both weight-lossdependent effects (such as decreased mechanical force on the neck, upper airway and diaphragm) and weightloss-independent effect due to metabolic changes (such as bile flow alteration, reduction of gastric size, anatomical rearrangement, vagal manipulation, and enteric gut hormone modulation) [24].

Recent emerging evidence suggests a positive outcome of bariatric surgery on cancer incidence in comparison to obese patients who did not undergo surgery [25]. A recent meta-analysis indicated a more pronounced protective effect of the bariatric procedure in women ( $\mathrm{HR}=0.58,95 \% \mathrm{CI}: 0.44-0.77)$ than in men $(\mathrm{HR}=0.97$, 95\% CI: 0.62-1.52) [25]. Weight loss itself, even without surgical intervention, has been demonstrated to decrease the risk of cancer. The etiology of this effect is complex, and it evolves inter alia from hormonal and inflammatory pathway alteration due to adipose tissue reduction [26].

\section{Medical management after bariatric surgery}

All patients after bariatric surgery require long-lasting observation for complications of bariatric procedures. Complementary follow-up pathways (surgical, medical, psychological, dietary) should be provided to all patients. Treatment outcome is primarily dependent on patient compliance. Patients should be aware not only of the necessity of a nutritionally adequate diet but also of the need for regular follow-up. They should be encouraged to incorporate moderate aerobic physical activity of at least 150 minutes per week (preferably 300 minutes weekly) [7].

Medical follow-up should begin at least 1 month after bariatric surgery, followed by at least 1 visit every 3 months after the operation in the first post-surgical year, every 6 months in the second year and annually thereafter [8]. Complete blood count, creatinine, liver function tests, glucose and electrolytes should be assessed during each visit. Serum concentration of iron, vitamin B12, vitamin 25(OH)D3, folate, calcium, parathormone and albumin should be performed before every type of surgery, and 6 months, 12 months and annually after RYGB, BPD and bilio-pancreatic diversion with a duodenal switch (BPD-DS) [27]. Optionally, screening for vitamin and trace element deficiency may be conducted (including vitamin B1, A, E, zinc, selenium and copper), but in the case of evidence of a poor diet or vomiting or malaise it should be checked.

\section{Early and late complications of bariatric surgery}

Post-bariatric complications can be considered as early or late. Early problems involving mainly anastomotic leakage, bleeding and pulmonary embolism are usually managed by the operating team during surgery or in the early post-operative period. The incidence of anastomotic leakage ranges from 0.1 to $5.6 \%$ [3], but the mortality rate is high and may reach $50 \%$, and therefore it is considered as the most life-threatening post-bariatric complication. Surgical exploration and treatment is needed if there is any clinical suspicion which may present as tachycardia, tachypnoea, hypoxia, increasing pain, fever or elevation of inflammatory markers [8].

In order to avoid deep vein thrombosis/pulmonary embolism, all bariatric patients should receive subcutaneous low-molecular weight heparin administration after surgery, and post-operatively until they are fully mobile. If possible, they should also be provided with graded compression stockings or intermittent pneumatic leg compression [8].

Late complications of bariatric surgery may be less urgent, but can still pose important health risks to patients. Some of the main issues relate to weight regain prevention, 
nutritional management, management of type 2 diabetes mellitus and lipid disorders, bone mineral metabolism and psychological disturbances. Vigilance for late complications from surgery (e.g. stricture) also remains necessary.

\section{Diabetes mellitus and lipid metabolism management}

In order to minimize the risk of hypoglycemia in T2DM patients, anti-diabetic pharmacotherapy should be adjusted immediately after bariatric surgery. According to the Guidelines of the International Federation for the Surgery of Obesity and the European Association for the Study of Obesity, improvement of T2DM may be considered to have occurred if:

- the post-operative insulin dose is reduced by at least $25 \%$ compared with pre-operatively,

- the post-operative oral anti-diabetic treatment dose is reduced by at least $50 \%$ compared with pre-operatively,

- there is a post-operative reduction in $\mathrm{HbA}_{1 \mathrm{c}}$ of at least $0.5 \%$ within 3 months or it reaches $<7.0 \%$ [8].

The Endocrine Society recommends the following postoperative glycemic control targets [27]:

- $\mathrm{HbA}_{1 \mathrm{c}} \leq 7.0 \%$ with

- fasting blood glucose $\leq 110 \mathrm{mg} / \mathrm{dl}$ and

- postprandial glucose $\leq 180 \mathrm{mg} / \mathrm{dl}$.

Diabetes mellitus and lipid disorder management should be considered concomitantly.

A substantial improvement in the metabolic state may be defined as [9]:

- $\mathrm{HbA}_{1 \mathrm{c}}$ reduced by $>2.0 \%$,

- $\mathrm{LDL}<2.3 \mathrm{mmol} / \mathrm{l}$,

- blood pressure < 135/85 mmHg,

- reduced medication from pre-operative state. T2DM remission has been defined as:

- partial remission - hyperglycemia below diagnostic thresholds for diabetes $\left(\mathrm{HbA}_{1 \mathrm{c}}>6 \%\right.$, but $<6.5 \%$, fasting plasma glucose [FPG] 100-125 mg/dl) of at least 1 -year duration, no active pharmacological therapy or ongoing procedures,

- complete remission - normal glycemic measures $\left(\mathrm{HbA}_{1 \mathrm{c}}\right.$ normal range $\left.[<6 \%], \mathrm{FPG}<100 \mathrm{mg} / \mathrm{dl}\right)$, at least 1 -year duration, no active pharmacological therapy or ongoing procedures,

- prolonged remission - complete remission of at least 5-year duration [8].

If clinical resolution of type 2 diabetes is demonstrated after surgery, anti-diabetic medications should be withdrawn. However, patients must continue capillary blood glucose monitoring according to their pre-operative needs. If further anti-diabetic management is necessary, insulin secretagogues should be discontinued and insulin doses should be adjusted to minimize the risk of hypoglycemia [7]. Metformin therapy might be applied with the dose of $500 \mathrm{mg}$ at the time of discharge and it should be adjusted to glycemia subsequently.

Although there have been studies demonstrating recovery from albuminuria in patients after bariatric surgery, one report showed deterioration of diabetes neuropathy and retinopathy after RYGB [28]. Therefore T2DM patients should be carefully observed on a regular basis for microvascular complications.

\section{Metabolic bone disease management}

Although not well recognized, obesity and osteoporosis coexist; loss of body mass profoundly influences bone metabolism. Vitamin D and calcium deficiencies with secondary hyperparathyroidism are common findings in extremely obese pre-bariatric surgery patients. Approximately $90 \%$ of obese individuals may be at risk of vitamin $\mathrm{D}$ deficiency (vitamin $\mathrm{D}$ concentration $<30 \mathrm{ng} / \mathrm{ml}$ ) prior to the bariatric procedure. Parathormone (PTH) levels are positively correlated with BMI, and $25-48 \%$ of obese individuals have an elevated PTH concentration [29]. Up to $25 \%$ of obese patients who have not undergone bariatric surgery have a pre-existing subclinical calcium deficiency (elevated PTH with normal $\mathrm{Ca}^{2+}$ ) [30]. This constellation of abnormalities predisposes extremely obese patients to low bone mass and osteomalacia even before surgery. Afterwards, as patients lose weight, their $\mathrm{PTH}$ levels continue to increase and the 25-hydroxyvitamin concentration decreases.

Restrictive as well as malabsorptive procedures appear to place patients at high risk of metabolic bone disease as a consequence of restricted oral intake, inadequate calcium and vitamin D supply, and rapid weight loss. Inappropriate supplementation post-operatively results in a significant bone disease 8 weeks to 32 years after surgery [31].

Voluntary weight loss of about $10 \%$ leads to bone loss of around 1-2\% at all sites [31]. It appears that more than $70 \%$ of patients who have had a malabsorptive procedure may develop metabolic bone disease; significant bone resorption becomes evident as soon as 3 months after bariatric surgery independently of the type of the procedure [32]. Although the data regarding the influence of bariatric surgery on bone mineral density are inconsistent, a growing number of studies indicate a bone mineral density (BMD) decline. In a study conducted by Carrasco et al., 12 months after gastric bypass surgery total BMD significantly decreased by $3 \%$ [33].

Post-bariatric supplementation should provide 1200$1500 \mathrm{mg}$ of calcium citrate (not calcium carbonate) and $3000 \mathrm{IU}$ of vitamin D in order to prevent metabolic bone disease. However, if vitamin D deficiency below $20 \mathrm{ng} / \mathrm{ml}$ develops, the therapeutic dose should provide 700010000 IU per day (depending on body mass) or 50000 IU per week [34]. 
Bone mineral density should be assessed before bariatric surgery and monitored annually after the procedure [27]. However, it might be complicated due to the high body mass of bariatric patients and technical capabilities of the dual-energy X-ray absorptiometry (DEXA) table.

\section{Gastroenterological and eating behavior management}

\section{Vomiting}

Reduced capacity of the gastric pouch after restrictive procedures $(30-60 \mathrm{ml})$ results in significant limitation of the amount of food consumed. Nausea, regurgitation and vomiting are particularly likely when compliance with nutritional recommendations is disregarded. Thirty to $60 \%$ of post-bariatric patients report vomiting, mostly within the first few months after surgery, when patients are adapting to the small gastric pouch [27]. Alarming symptoms, such as persistent vomiting, nausea, abdominal pain, diarrhea and constipation, should lead to the careful further assessment of other overlapping disorders such as bacterial overgrowth, ulcer disease, anastomotic complications, and bowel obstruction.

\section{Dumping syndrome}

Rapid gastric emptying and early exposure of the small intestine to energy-dense nutrients may trigger a group of symptoms constituting dumping syndrome (DS). Early symptoms comprise gastrointestinal and vasomotor manifestations, while late DS stems from postprandial hypoglycemia [35].

Among other factors contributing to the pathogenesis of the syndrome there is a release of gastrointestinal and pancreatic hormones, such as enteroglucagon, vasoactive intestinal peptide, peptide YY, pancreatic polypeptide and neurotensin [36]. Key symptoms of early DS develop 15-30 minutes after a meal and include:

- gastrointestinal symptoms - abdominal pain, nausea, epigastric fullness, diarrhea, borborygmi,

- vasomotor manifestation - dizziness, flushing, dyspnea, tachycardia, apathy, weakness and syncope [27].

The prevalence of DS ranges between 40 and $76 \%$ of patients after RYGB. Although restrictive interventions such as SG should not be expected to exert DS, two prospective studies demonstrated development of symptoms suggestive of dumping syndrome 6-12 months after SG in up to $40 \%$ of patients [27,37-39]. Treatment of DS should be started with implementation of nutritional change. Patients should eat at least 3 small, frequent meals and avoid refined-carbohydrate dense food. They should chew small bites of food prior to swallowing, and liquids should not be ingested earlier than 30 minutes after a solid-food meal. Ingestion of protein, fiber and complex carbohydrates should be increased.
Pharmacological intervention includes administration of somatostatin analogues, such as octreotide $50 \mathrm{mg}$ s.c. - given 30 minutes prior to the meal it may improve symptoms in some patients [27]. A novel agent, undergoing phase 2 testing - pasireotide - may become an alternative for the pharmacological treatment of DS [40].

Symptoms of DS usually resolve spontaneously 18-24 months after surgery. Controversially, although considered as a complication, DS may be in fact a desired element of behavior modification. It discourages the intake of energy-dense food and beverages [27]. Nevertheless, it has been shown that better adherence to dietary advice from the very beginning in non-dumpers leads to a greater decrease in BMI at 1 and 2 years of follow-up [37].

\section{Postprandial hypoglycemia}

Postprandial hyperinsulinemic hypoglycemia with neuroglycopenic symptoms (such as altered mental status, loss of consciousness) has been observed in some patients after RYGB. It is frequently identified with dumping syndrome, although in distinction to hyperinsulinemic hypoglycemia, DS rarely presents with symptoms of neuroglycopenia [41]. Postprandial hypoglycemia tends to develop usually 2 to 9 years after gastric bypass [41]. Patients presenting neuroglycopenic symptoms, who do not respond to nutritional modification, should be evaluated to differentiate dumping syndrome, insulinoma and noninsulinoma pancreatogenous hypoglycemia syndrome (NIPHS) [42,43]. Noninsulinoma pancreatogenous hypoglycemia syndrome was introduced for the first time by Service et al. in 1999 [42]. Suggested mechanisms have ranged from expansion of beta cell mass (e.g. nesidioblastosis) to alterations in beta cell function (glucagon-like peptide- 1 contributes to beta cell proliferation) and non-beta cell related factors (decreased levels of the appetite-stimulating and insulin counter-regulatory gastrointestinal hormone), or possibly a combination of mechanisms [43]. Diagnostic criteria for NIPHS are presented in Table 1.

Treatment options of NIPHS include:

- the somatostatin analogue octreotide, as it inhibits gastric emptying, gastrointestinal hormones, and insulin secretion [27],

- acarbose, which improves symptoms due to reduction of carbohydrate absorption,

- diazoxide - a calcium-induced insulin release inhibitor.

Some authors suggest partial pancreatectomy as a treatment alternative for patients who do not improve after pharmacological treatment, but this strategy is controversial, and it should be reserved for the rare severe cases resistant to medical measures [43]. 
Table 1. Diagnostic criteria for noninsulinoma pancreatogenous hypoglycemia syndrome [42]

\begin{tabular}{|l|l|}
\hline Criterion element & Comment \\
\hline 1. Positive Whipple's triad & $\begin{array}{l}\text { - Episodic hypoglycemia } \\
\text { - Central nervous system dysfunction temporally related to hypoglycemia } \\
\text { (confusion, anxiety, stupor, paralysis, convulsions, coma) } \\
\text { - Reversal of these abnormalities by glucose administration }\end{array}$ \\
\hline 2. A negative 72-hour fast & - Negative peri-operative radiographic imaging \\
\hline 3. Negative observation toward insulinoma & $\begin{array}{l}\text { - Nesidioblastosis - } \beta \text {-cell hyperplasia and diffuse proliferation and } \\
\text { hypertrophy of islet cells from pancreatic ducts }\end{array}$ \\
\hline 4. Positive calcium stimulation test & \\
\hline 5. Islet hypertrophy or nesidioblastosis
\end{tabular}

\section{Management of hepatobiliary complications}

Cholelithiasis is a common complication of rapid weight loss, occurring in $13-36 \%$ of patients within 6 months after RYGB, but also after SG [44]. Cholecystectomy at the time of bariatric surgery is still a matter of debate. Some specialists suggest performing routine cholecystectomy at the time of gastric bypass, especially considering the technical difficulties in performing endoscopic retrograde cholangiopancreatography after RYGB [44]. However, most surgeons perform cholecystectomy only if the patient is symptomatic [45]. A possible approach is administration of ursodeoxycholic acid (UDA) post-operatively. According to a double blind prospective randomized trial by Sugerman et al. [46] a daily dose of $600 \mathrm{mg}$ UDA is an effective prophylaxis for gallstone formation.

\section{Pharmacotherapy remarks}

For the first 4 weeks following the surgery, crushed or liquid medications should be administered in rapidrelease, non-enteric-coated formulations to maximize absorption [8]. If drug levels can be measured (e.g. anticonvulsants, lithium) or effects monitored (e.g. thyroid hormone replacement), it may be reasonable to make checks 1-2 months after surgery. For the first 6 months following the RYGB and SG procedure, therapy with proton pump inhibitors is recommended in order to prevent ulceration at the pouch jejunostomy.

\section{Hypoglycemic treatment}

Metformin is a first-line drug in obese diabetic patients. Since metformin is absorbed mainly in the proximal part of the small intestine, the question of its post-bariatric surgery pharmacokinetics has been raised. Contrary to the hypothesis that absorption would be significantly impaired in patients after RYGB, the only study conducted to date demonstrated that the absorption and bioavailability of metformin in these patients is increased [47]. Further studies are required in order to establish the clinical significance of this finding.

\section{Antihypertensive agents and statins}

The need for antihypertensive drugs, including diuretics, is reduced by around 50\% after bariatric surgery [48].

Although antihypertensive drugs of all groups are frequently used in bariatric surgery patients, only studies on the pharmacokinetics of beta blockers (propranolol and atenolol) have been conducted. Due to the changes in pharmacokinetics of propranolol after partial gastrectomy (probably because of its lipophilicity), hydrophilic drugs e.g. atenolol may be preferred, if beta blocker therapy is still necessary [49].

Available evidence shows that bariatric surgery is effective for decreasing the use of statins for dyslipidemia. Post-operatively patients on statin treatment should be retitrated on the lowest dose possible while monitoring the serum lipids profile [50].

\section{Oral contraceptives}

According to the reproductive hormone profile and pregnancy outcomes, fertility status improves rapidly after bariatric surgery [51]. However, women who have undergone a bariatric procedure should be aware of possibly lower efficacy of contraceptives, due to malabsorption and post-operative complications, such as diarrhea and vomiting. Patients should not rely on combined oral contraceptives (COC) and if menstrual (as is the case immediately after bariatric surgery) should consider dual contraception (e.g. condom + COC).

\section{Behavioral and psychological support}

Psychological assessment is an essential part of preoperative evaluation indentifying potential contraindications for bariatric surgery or post-operative risks. Nonstabilized psychotic disorders, severe depression and personality disorders are contraindications for bariatric 
surgery [8]. Whether patients with active eating behavior disorders should undergo surgery is still a matter of debate [52]. Some authors suggest that binge eating may be alleviated after the bariatric procedure, mostly because consumption of unusually large meals becomes physically impossible. However, a recent study on eating behavior 2 years after bariatric surgery provided data about a subgroup of patients who reported vomiting for weight and shape reasons [53].

Post-operative psychological care is as vital as other surveillance aspects. A number of studies have shown postoperative improvement in depressive symptoms, self-esteem, health-related quality of life, and body image [54]. However, a lot of patients continue to suffer from emotional disturbances, mainly due to unrealistic pre-surgical expectations concerning weight loss and difficulties associated with the necessity of post-bariatric life style changes. Several studies have reported increased suicide incidence in patients after bariatric surgery, with an estimated suicide rate of 3.9/10 000, compared with $1.0 / 10000$ in the general population [55]. The most common reason found is depression, but the spectrum of mental disorders linked to suicidal ideation and attempts is wide. A lifetime prevalence of $30 \%$ for mood disorders, $23 \%$ for substance abuse and $48 \%$ for anxiety disorders was revealed in morbidly obese bariatric patients [56]. Difficulties in coping with lifestyle changes, disappointment about failed weight loss or weight regain, and recurrence of obesity-related diseases after initial improvement are additional burdens in this group. According to Tindle et al., $70 \%$ of patients who committed suicide did so within 3 years after the bariatric procedure [57]. Therefore, post-bariatric treatment should be provided for as long as the patient demonstrates psychological distress or has difficulties in maintaining behavioral changes and the diet regime [58].

\section{Conclusions}

Even though bariatric surgery is the most effective method of achieving long-lasting weight loss and improving metabolic and cardiovascular outcomes in selected patients, not everyone is eligible or meets the criteria for surgery. After all, bariatric surgery should be considered as a method of ultima ratio after exhausting all other medical possibilities. If performed, multidisciplinary surveillance is important for maintaining optimal health of patients in the long term. One of the most important aspects of post-bariatric follow-up is nutritional care and eating behavior modification. Severe nutritional deficits can be avoided after bariatric procedures, provided patients are systematically supplemented with multivitamins and carefully monitored. Similarly, the majority of post-surgical complications may be prevented if patients are aware of the fact that surgical treatment is inseparably associated with lifelong behavioral changes.

\section{Disclosure}

Gabriela Handzlik-Orlik was supported by the European Community from the European Social Fund within the DoktoRIS project.

Other authors report no conflict of interest.

\section{References}

1. Pêgo-Fernandes PM, Bibas BJ, Deboni M. Obesity: the greatest epidemic of the 21st century? Sao Paulo Med J 2011; 129: 283-284.

2. Finkelstein EA, Khavjou OA, Thompson $\mathrm{H}$, et al. Obesity and severe obesity forecasts through 2030. Am J Prev Med 2012; 42: 563-570.

3. Baptista V, Wassef W. Bariatric procedures: an update on techniques, outcomes and complications. Curr Opin Gastroenterol 2013; 29: 684-693.

4. Neff KJ, le Roux CW. Bariatric surgery: a best practice article. J Clin Pathol 2013; 66: 90-98.

5. Sjöström L, Peltonen M, Jacobson P, et al. Bariatric surgery and long-term cardiovascular events. JAMA 2012; 307: 56-65.

6. Yousseif A, Emmanuel J, Karra E, et al. Differential effects of laparoscopic sleeve gastrectomy and laparoscopic gastric bypass on appetite, circulating acyl-ghrelin, peptide YY3-36 and active GLP-1 levels in non-diabetic humans. Obes Surg 2014; 24: 241-252.

7. Mechanick J, Youdim A, Jones DB, et al. Clinical practice guidelines for the perioperative nutritional, metabolic, and nonsurgical support of the bariatric surgery patient - 2013 update: cosponsored by American Association of Clinical Endocrinologists, the Obesity Society, and American Society for Metabolic \& Bariatric Surgery. Surg Obes Relat Dis 2013; 9: 159-191.

8. Fried M, Yumuk V, Oppert JM, et al. Interdisciplinary European guidelines on metabolic and bariatric surgery. Obes Facts 2013; 6: 449-468.

9. Dixon JB, Zimmet P, Alberti KG, et al. International Diabetes Federation Taskforce on Epidemiology and Prevention. Bariatric surgery: an IDF statement for obese Type 2 diabetes. Arq Bras Endocrinol Metabol 2011; 55: 367-382.

10. Gloy VL, Briel M, Bhatt DL, et al. Bariatric surgery versus non-surgical treatment for obesity: a systematic review and meta-analysis of randomised controlled trials. BMJ 2013; 347: f5934.

11. Sjöström L, Lindroos AK, Peltonen M, et al; Swedish Obese Subjects Study Scientific Group. Lifestyle, diabetes, and cardiovascular risk factors 10 years after bariatric surgery. N Engl J Med 2004; 351: 2683-2693.

12. Levy P, Fried M, Santini F, et al. The comparative effects of bariatric surgery on weight and type 2 diabetes. Obes Surg 2007; 17: 1248-1256.

13. Sjöström L. Review of the key results from the Swedish Obese Subjects (SOS) trial - a prospective controlled intervention study of bariatric surgery. J Intern Med 2013; 273: 219-234.

14. DiGiorgi M, Rosen DJ, Choi JJ. Re-emergence of diabetes after gastric bypass in patients with mid- to long-term follow-up. Surg Obes Relat Dis 2010; 6: 249-253.

15. Ashrafian H, le Roux CW, Darzi A, et al. Effects of bariatric surgery on cardiovascular function. Circulation 2008; 118: 2091-2102. 
16. Buchwald H, Avidor Y, Braunwald E, et al. Bariatric surgery: a systematic review and meta-analysis. JAMA 2004; 292: 1724-1737.

17. Bolignano D, Zoccali C. Effects of weight loss on renal function in obese CKD patients: a systematic review. Nephrol Dial Transplant 2013; 28: 82-98.

18. Navaneethan SD, Yehnert H. Bariatric surgery and progression of chronic kidney disease. Surg Obes Relat Dis 2009; 5 : 662-665.

19. Navarro-Díaz M, Serra A, Romero R, et al. Effect of drastic weight loss after bariatric surgery on renal parameters in extremely obese patients: long-term follow-up. J Am Soc Nephrol 2006; 17: S213-217.

20. Fraile-Gómez P, Domínguez-Quibén S, Sebastía-Morant J, et al. Bariatric surgery, a new cause of acute renal failure. Nefrologia 2013; 33: 616-618.

21. Nagaraju SP, Gupta A, McCormick B. Oxalate nephropathy: an important cause of renal failure after bariatric surgery. Indian J Nephrol 2013; 23: 316-318.

22. Weingarten TN, Gurrieri C, McCaffrey JM. Acute kidney injury following bariatric surgery. Obes Surg 2013; 23: 64-70.

23. Ravesloot MJ, van Maanen JP, Hilgevoord AA, et al. Obstructive sleep apnea is underrecognized and underdiagnosed in patients undergoing bariatric surgery. Eur Arch Otorhinolaryngol 2012; 269: 1865-1871.

24. Sarkhosh K, Switzer NJ, El-Hadi M, et al. The impact of bariatric surgery on obstructive sleep apnea: a systematic review. Obes Surg 2013; 23: 414-423.

25. Tee MC, Cao Y, Warnock GL, et al. Effect of bariatric surgery on oncologic outcomes: a systematic review and meta-analysis. Surg Endosc 2013; 27: 4449-4456.

26. Byers T, Sedjo RL. Does intentional weight loss reduce cancer risk? Diabetes Obes Metab 2011; 13: 1063-1072.

27. Heber D, Greenway FL, Kaplan LM, et al. Endocrine Society. Endocrine and nutritional management of the post-bariatric surgery patient: an Endocrine Society Clinical Practice Guideline. J Clin Endocrinol Metab 2010; 95: 4823-4843.

28. Chuah LL, le Roux CW. Management of patients with type 2 diabetes before and after bariatric surgery: evolution and microvascular complications. Nutr Hosp 2013; 28 Suppl 2: 17-22.

29. Carlin AM, Rao DS, Meslemani AM, et al. Prevalence of vitamin D depletion among morbidly obese patients seeking gastric bypass surgery. Surg Obes Relat Dis 2006; 2: 98-103.

30. Hamoui N, Anthone G, Crookes PF. Calcium metabolism in the morbidly obese. Obes Surg 2004; 14: 9-12.

31. Williams SE, Cooper K, Richmond B, et al. Perioperative management of bariatric surgery patients: focus on metabolic bone disease. Cleve Clin J Med 2008; 75: 333-334, 336, 338 passim.

32. Coates PS, Fernstrom JD, Fernstrom MH, et al. Gastric bypass surgery for morbid obesity leads to an increase in bone turnover and a decrease in bone mass. J Clin Endocrinol Metab 2004; 89: 1061-1065.

33. Carrasco F, Ruz M, Rojas P, et al. Changes in bone mineral density, body composition and adiponectin levels in morbidly obese patients after bariatric surgery. Obes Surg 2009; 19: 41-46.

34. Płudowski P, Karczmarewicz E, Bayer M, et al. Practical guidelines for the supplementation of vitamin $\mathrm{D}$ and the treatment of deficits in Central Europe - recommended vitamin D intakes in the general population and groups at risk of vitamin D deficiency. Endokrynol Pol 2013; 64: 319-327.

35. Tack J, Arts J, Caenepeel P, et al. Pathophysiology, diagnosis and management of postoperative dumping syndrome. Nat Rev Gastroenterol Hepatol 2009; 6: 583-590.

36. Tack J, Deloose E. Complications of bariatric surgery: Dumping syndrome, reflux and vitamin deficiencies. Best Pract Res Clin Gastroenterol 2014; 28: 741-749.
37. Banerjee A, Ding Y, Mikami DJ, et al. The role of dumping syndrome in weight loss after gastric bypass surgery. Surg Endosc 2013; 27: 1573-1578.

38. Tzovaras G, Papamargaritis D, Sioka E, et al. Symptoms suggestive of dumping syndrome after provocation in patients after laparoscopic sleeve gastrectomy. Obes Surg 2012; 22: 23-28.

39. Papamargaritis D, Koukoulis G, Sioka E, et al. Dumping symptoms and incidence of hypoglycaemia after provocation test at 6 and 12 months after laparoscopic sleeve gastrectomy. Obes Surg 2012; 22: 1600-1606.

40. Deloose E, Bisschops R, Holvoet L, et al. A pilot study of the effects of the somatostatin analog pasireotide in postoperative dumping syndrome. Neurogastroenterol Motil 2014; 26: 803-809.

41. Bernard B, Kline GA, Service FJ. Hypoglycaemia following upper gastrointestinal surgery: case report and review of the literature. BMC Gastroenterol 2010; 10: 77.

42. Service FJ, Natt N, Thompson GB, et al. Noninsulinoma pancreatogenous hypoglycemia: a novel syndrome of hyperinsulinemic hypoglycemia in adults independent of mutations in Kir6.2 and SUR1 genes. J Clin Endocrinol Metab 1999; 84: 1582-1589.

43. Foster-Schubert KE. Hypoglycemia complicating bariatric surgery: incidence and mechanisms. Curr Opin Endocrinol Diabetes Obes 2011; 18: 129-133.

44. Villegas L, Schneider B, Provost D, et al. Is routine cholecystectomy required during laparoscopic gastric bypass? Obes Surg 2004; 14: 206-211.

45 Li VK, Pulido N, Martinez-Suartez P, et al. Symptomatic gallstones after sleeve gastrectomy. Surg Endosc 2009; 23: 2488-2492.

46. Sugerman HJ, Brewer WH, Shiffman ML, et al. A multicenter, placebo-controlled, randomized, double-blind, prospective trial of prophylactic ursodiol for the prevention of gallstone formation following gastric-bypass-induced rapid weight loss. Am J Surg 1995; 169: 91-96.

47. Padwal RS, Gabr RQ, Sharma AM, et al. Effect of gastric bypass surgery on the absorption and bioavailability of metformin. Diabetes Care 2011; 34: 1295-1300.

48. Hodo DM, Waller JL, Martindale RG, et al. Medication use after bariatric surgery in a managed care cohort. Surg Obes Relat Dis 2008; 4: 601-607.

49. Wójcicki J, Wojciechowski G, Wójcicki M, et al. Pharmacokinetics of propranolol and atenolol in patients after partial gastric resection: a comparative study. Eur J Clin Pharmacol 2000; 56: 75-79.

50. Yska JP, van der Linde S, Tapper VV, et al. Influence of bariatric surgery on the use and pharmacokinetics of some major drug classes. Obes Surg 2013; 23: 819-825.

51. Gerrits EG, Ceulemans R, van Hee R, et al. Contraceptive treatment after biliopancreatic diversion needs consensus. Obes Surg 2003; 13: 378-382.

52. Niego SH, Kofman MD, Weiss JJ, et al. Binge eating in the bariatric surgery population: a review of the literature. Int J Eat Disord 2007; 40: 349-359.

53. de Zwaan M, Hilbert A, Swan-Kremeier L, et al. Comprehensive interview assessment of eating behavior 18-35 months after gastric bypass surgery for morbid obesity. Surg Obes Relat Dis 2010; 6: 79-85.

54. Karlsson J, Sjöström L, Sullivan M. Swedish obese subjects (SOS) - an intervention study of obesity. Two-year follow-up of health-related quality of life (HRQL) and eating behavior after gastric surgery for severe obesity. Int J Obes Relat Metab Disord 1998; 22: 113-126.

55. Peterhänsel C, Petroff D, Klinitzke G, et al. Risk of completed suicide after bariatric surgery: a systematic review. Obes Rev 2013; 14: 369-382. 
56. Scholtz S, Bidlake L, Morgan J, et al. Long-term outcomes following laparoscopic adjustable gastric banding: postoperative psychological sequelae predict outcome at 5-year follow-up. Obes Surg 2007; 17: 1220-1225.

57. Tindle HA, Omalu B, Courcoulas A, et al. Risk of suicide after long-term follow-up from bariatric surgery. Am J Med 2010; 123: 1036-1042.

58. Kubik JF, Gill RS, Laffin M, et al. The impact of bariatric surgery on psychological health. J Obes 2013; 2013: 837989. 